\title{
Desktop 3D Laser Scanning Technology Research
}

\author{
Jiajun $\operatorname{Lin}^{\mathrm{a}}$, Daxuan $\mathrm{Hu}^{* \mathrm{~b}}$, Hong Chen* \\ School of Mechanical and Electric Engineering, Laboratory Center, \\ Guangzhou University, \\ Guangzhou, China, 510006

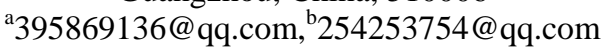

\begin{abstract}
This passage puts forward a kind of simple and convenient three-dimensional laser measurement technology for a small volume and complex shape space data acquisition of the object. This technology mainly uses the camera self calibration method for construction of three-dimensional model algorithm and creates the measurement environment of the measuring technique in machinery and hardware camp. The result of the experiment shows that the desktop 3D laser scanning measuring instrument is convenient, and can acquire spatial location ideal data with low cost.

For the measurement of small zero, components are obtained by the traditional measuring instruments. The measurement of the traditional method has many disadvantages such as the object of measuring confined to regular shape and the measurement is more troublesome, time-consuming and laborious. However, 3D laser scanner technology can finish measurement of complex objects. Its advantage is high precision, high speed, especially time and cost saving. This technology is mainly aimed at some smaller objects and provides a method for quick access to the object of spatial data. Currently on the market there are also some mature 3D scanning instruments, such as the Fastscan, but the price is expensive, normally costing hundreds of thousands of dollars. Some small businesses can't afford it at all.
\end{abstract}

Keywords-desktop; 3D laser scanner; the camera self calibration method

\section{The Measuring Principle Of 3D LASER ScAnNer}

The measuring principle of $3 \mathrm{~d}$ laser scanner is through the laser to the measured object, and camera by the laser falls on the entity space distance and into space. Objective table rotates, camera for 360 degrees the space position of the object to be tested can complete access to the entire space location data of the object, thus generating space model of the object to be tested

\section{THE MEASUREMENT MODEL OF 3D LASER SCANNER}

This paper studies the 3D laser scanner which is composed of five parts, the stage, and laser scanning station, camera, LED spotlights and a box body. As shown in figure 1, the object stage measured by the control system automatically, draw a circle after each measurement. The absolute value encoder accurately read the angle of rotation and the mechanical devices are ensuring to 360 degrees in accuracy. The error is controlled in 0.01 degrees. During the turntable rotation time, the camera keep photographing constantly then the spatial location of objects can be obtained in different directions. Through the control of upper and lower computer, to ensure the synchronous turntable and a camera obtains photos synchronization. Because this technique obtains the object space data in scanning needs to be measured in light and dark in two environments paired check results, so it needs to provide sealed box to create complete darkness. The box is installed with LED spotlight providing light environment. And then through the set of the program and circuit, the 3D laser scanner can automatically scan.

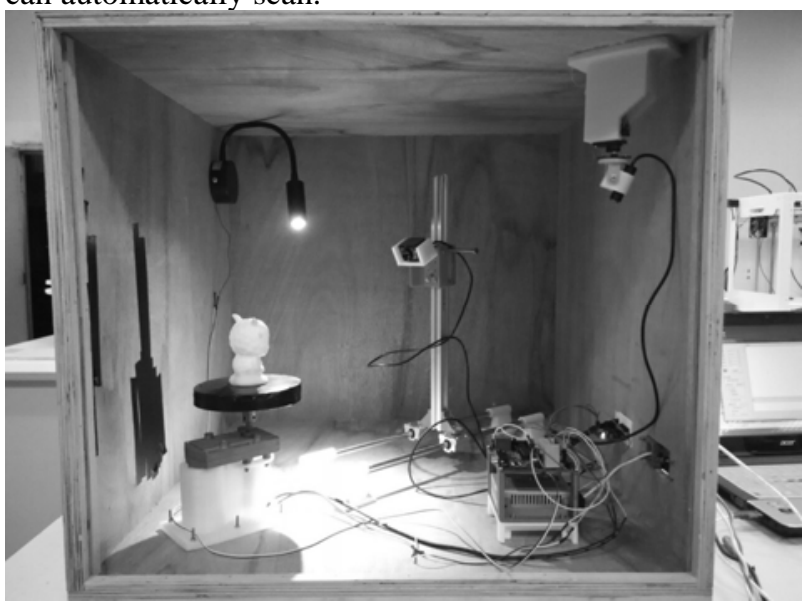

Figure.1The measurement model of 3D laser scanner 


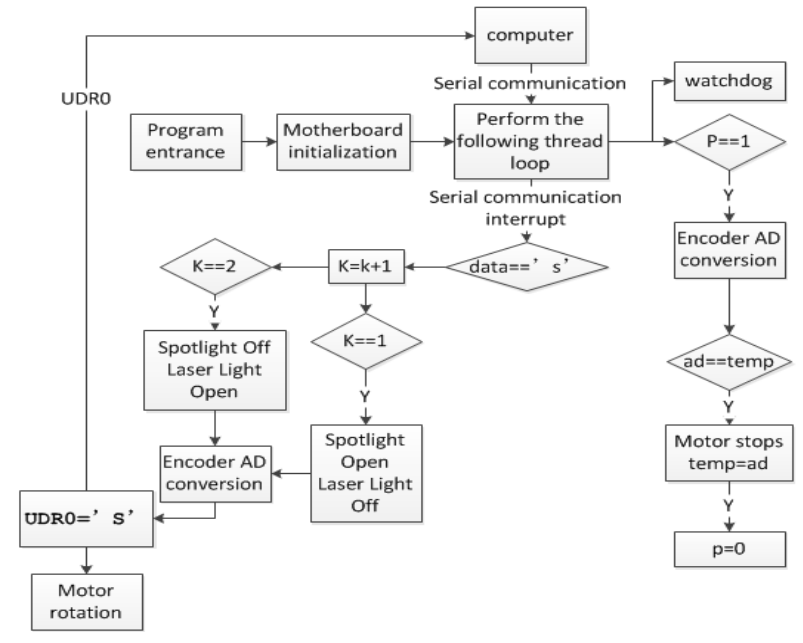

Figure 2. The lower computer work flowchart

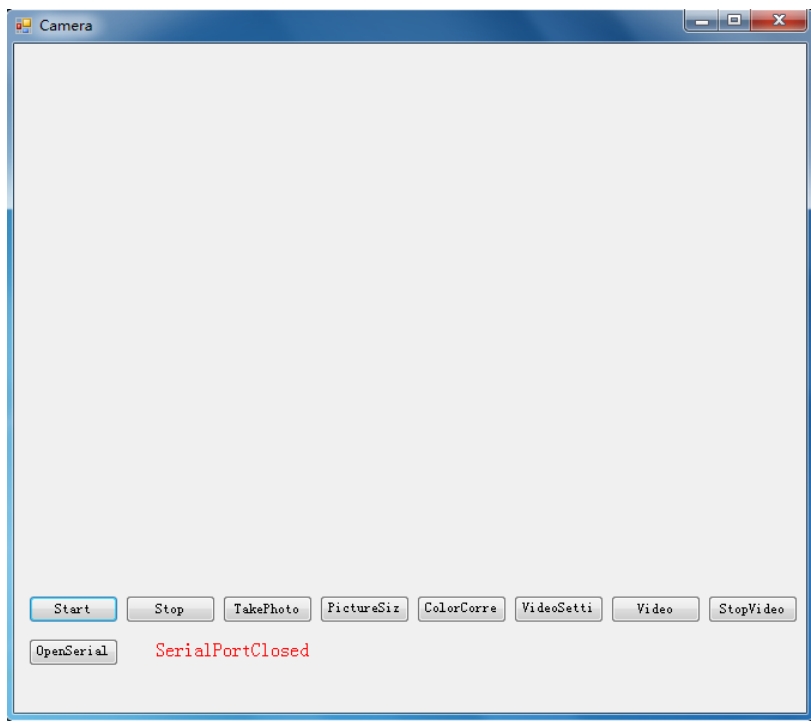

Figure 3. The upper computer software interface

\section{DESKTOP 3D LASER SCANNER WORK FLOW CHART}

Working process of 3D scanner. First initialize each port, and then enter the circulation. When the upper computer through the serial port to send the character's' to the lower machine, and the spotlight under control is open. And then through the serial port returns the character's' to the host computer, the upper computer after receiving information started taking pictures. At the same time, motor rotation stops a week under a machine control motor rotation. After taking the pictures in the light of circumstances, through the PC to MCU sends the command, the lower machine will be photographed environment and switch to the laser pen work situations (Spotlight doesn't work at this time). Through QT program to deal with images, it will get 200 images, generating a file types, reoccupying mash lab open, observing the generated $3 \mathrm{~d}$ model. The lower computer work flowchart is shown in figure 2 .The upper computer software interface is shown in figure 3.

\section{CONSTRUCTION OF THREE-DIMENSIONAL MODEL ALGORITHM}

Obtaining the spatial data of the object must be marked to identify the location of spatial position, there are several ways constructing three-dimensional model algorithm, such as: camera self calibration method, laser center extraction method, acquisition point cloud method, point cloud registration method. On the basic of test validate, the camera calibration method is most suitable for this project. Camera self calibration doesn't need calibration block; it only gets through the relationship between the image point of the process of camera calibration. It appears because of the actual application requirements and replying forum shifting. The features of the self calibration method is relying on the correspondence relationship between the multiple images of the calibration. It has many advantages, for example, it only needs to establish a correspondence between images and it is very flexible. it also has a wide range of potential applications.

Self calibration is the intrinsic parameter calibration matrix K camera

$K=\left(\begin{array}{ccc}f_{u} & s & u_{0} \\ 0 & f_{v} & v_{0} \\ 0 & 0 & 1\end{array}\right)$

If two homogeneous coordinates differ a nonzero factor, then the two coordinates has the same homogeneous.

The camera model expression is:

$$
\begin{gathered}
m \approx P_{l}^{E}\left(\begin{array}{c}
X \\
1
\end{array}\right), \quad m^{\prime} \approx P_{r}^{E}\left(\begin{array}{c}
X \\
1
\end{array}\right) \\
m \approx K X, \quad m^{\prime} \approx K(R X+T), \quad T \otimes\left(K^{-1} m^{\prime}\right) \approx T \otimes R X \\
\left(m^{\prime}\right)^{T} K^{-T}[T]_{\times} R K^{-1} m=0, \quad\left(m^{\prime}\right)^{T} F m=0 \\
F \approx K^{-T}[T]_{\times} R K^{-1}
\end{gathered}
$$

The rank of $\mathrm{F}$ is $2 . \mathrm{F}$ is certain under the difference between a constant factor. $\mathrm{F}$ can be determined by 8 corresponding points on the image of linear. The calibration results:

\section{EXPERIMENTAL RESULTS AND ANALYSIS}

Through simulation and experiment, verified the effectiveness of the 3D laser scanner model parameters of the proposed calibration algorithm. Do scanning test through a doll and the experimental result shown in 3D QT software. The comparison of real object and generated 3D model are as follows: 


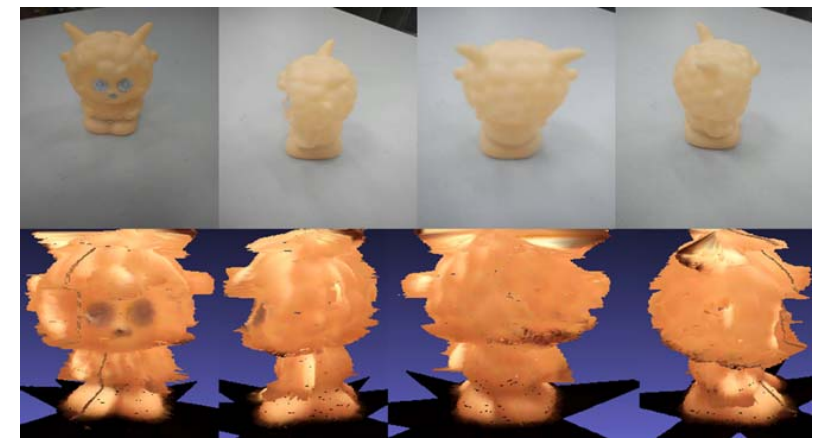

$$
\mathrm{K}=\left(\begin{array}{ccc}
409.3178 & 0 & 326.9076 \\
0 & 408.6694 & 322.9966 \\
0 & 0 & 1.0000
\end{array}\right)
$$

Analysis. The experimental results show that 3D model of scanning and the real object is very close, achieving the reduction effect. But there exists little error, generating excess burrs. The main reasons are as follows. Firstly, the start position of the motor causes little error; the rotational speed can't guarantee completely consistent between the first time in light and the second time without light. So it is necessary to replace the more motor with higher accuracy and then improve the control circuit and stable the power supply.

\section{CONClusions}

This paper, based on the camera self calibration algorithm, presents a technology for convenient access to smaller volume, more complicated shape object's spatial data. The experiment result shows that 3D model we have got is basically consistent with the real one and we made it with short scanning acquisition time, high precision, low cost. Desktop 3D laser scanner has a wide application prospect and using value in reverse engineering, which can shorten the product development cycle, improve economic benefit. This instrument laid a solid foundation for the realization of the popular promotion.

\section{ACKNOWLEDGMENTS}

We gratefully acknowledge the financial support by Chen Hong.

\section{REFERENCES}

[1] Tsai R.Y.An Efficient and accurate camera calibration technique for 3D machine vision[C].In:Proc IEEE Confern Computer Vision and Pattern Recognition, 1986: 364-374.

[2] Tsai R. A versatile camera calibration technique for high-accuracy 3D machine vision metrology using off-the-shelf TV cameras and lenses[J]. Robotics and Automation, IEEE Journal of, 1987, 3(4): 323-344.

[3] Fu Pei. Study on Key Technologies of low-cost 3D laser scanner system [D]. Harbin Institute of Technology, 2010.

[4] Md. Atiqur Rahman Ahad. Computer Vision and Action Recognition - A Guide for Image Processing and Computer Vision Community for Action Understanding[M], 2011.

[5] Deng Chunmei, Chen Jihong, Zhou Huicheng, et al. [J]. journal, calibrating line structured laser measurement system of Huazhong University of science and technology 2000, 28 (03): 19-21.

[6] Zhou Huicheng, Chen Jihong, Zhou Ji. The calibration method of basic parameters of a new head of line structured light vision sensing [J]. Chinese Journal of scientific instrument, 2000,21 (2): 125-131.

[7] Heikkila J, Silven O. A four-step camera calibration procedure with implicit image correction[C]//Computer Vision and Pattern Recognition, 1997. Proceedings., 1997 IEEE Computer Society Conference on. IEEE, 1997: 1106-1112. 